\title{
Are computerised respiratory sounds in COPD gender dependent?
}

\author{
C. Jácome \\ Research Centre in Physical Activity, Health and Leisure (CIAFEL), Faculty of Sports, University of Porto, \\ Porto, Portugal \\ Lab $3 R$ - Respiratory Research and Rehabilitation Laboratory, School of Health Sciences, University of \\ Aveiro (ESSUA), Aveiro, Portugal
}

\section{A. Marques}

Lab $3 R$ - Respiratory Research and Rehabilitation Laboratory, School of Health Sciences, University of Aveiro (ESSUA), Aveiro, Portugal

Center for Health Technology and Services Research (CINTESIS), School of Health Sciences, University of Aveiro, Aveiro, Portugal

ABSTRACT: This study explored gender differences in normal and adventitious respiratory sounds (RS) of patients with COPD. Twenty-six patients were enrolled. RS were recorded simultaneously at posterior right/left chest and airflow standardised (0.4-0.61/s). Breathing phases were automatically detected using the airflow signals. Normal RS, crackles and wheezes were analysed with developed algorithms. The frequency of maximum intensity and the mean intensity of inspiratory normal RS were slightly higher in females than in males. During expiration, the mean intensity was lower in females, however, a significant difference was only found at posterior left $(\mathrm{p}=0.01)$. The mean number of crackles and wheezes seemed to be higher in male patients, although a significant difference was only observed in expiratory crackles at posterior right chest $(\mathrm{p}=0.04)$. Findings suggest that minor differences exist between female and male patients with COPD regarding normal and adventitious RS. However, it is still unknown if these differences are clinically relevant.

\section{INSTRUCTION}

Acute exacerbations are one of the most important causes of morbidity and mortality in patients with Chronic Obstructive Pulmonary Disease (COPD) (Vestbo et al., 2013). Therefore, methods to early diagnose acute exacerbations of COPD (AECOPD) are of paramount importance worldwide.

Currently, the diagnosis of an AECOPD relies exclusively on the clinical presentation of the patient reporting an acute change of symptoms (baseline dyspnoea, cough and/or sputum production), that is beyond normal day-to-day variation (Vestbo et al., 2013). This may affect the timely diagnosis of exacerbation events and patients' optimal management.

During an AECOPD there is increased hyperinflation and gas trapping (Vestbo et al., 2013) and it has been shown that computerised respiratory sounds (RS), namely normal and adventitious RS, are directly related to movement of air and presence of secretions (Bohadana et al., 2014). Thus, computerised RS may have the potential to monitor respiratory status in patients with COPD and to diagnose AECOPD more objectively.

Computerised auscultation has shown that in stable patients with COPD, normal RS characteristics are similar to those observed in healthy people and adventitious RS are mainly characterised by inspiratory crackles and expiratory wheezes (Jácome \& Marques, 2015). However, while in healthy people it is recognised that $\mathrm{RS}$ are affected by gender
(Gavriely et al., 1995), in patients with COPD this is still unknown.

This study explored gender differences in normal and adventitious RS of patients with COPD.

\section{MATERIALS AND METHODS}

\subsection{Study design and Participants}

A cross-sectional study with female and male outpatients with COPD was conducted. Patients from both genders were included if they had a diagnosis of COPD according to the Global initiative for Chronic Obstructive Lung Disease (GOLD) (Vestbo et al., 2013). Patients with regular appointments with their general practitioner and clinically stable for 1 month prior to the study were eligible. Exclusion criteria were presence of co-existing respiratory diseases or severe neurological, musculoskeletal or psychiatric impairments. Approval for this study was obtained from the ethics committee of the Centre Health Regional Administration. Written informed consent was obtained prior to any data collection.

\subsection{Data collection procedures}

Socio-demographic (age and gender), anthropometric (body mass index) and clinical (modified British Medical Research Council questionnaire for dyspnoea (Vestbo et al., 2013)) data were first collected. 
Then, RS and airflow (pneumotachograph 3830, Hans Rudolph, Inc., Shawnee, KS, USA) were acquired simultaneously for 20-seconds. Patients were in a seated-upright position, wearing a nose clip and breathing through a mouthpiece at a typical tidal airflow $(0.4-0.6 \mathrm{~L} / \mathrm{s})$. Visual biofeedback of the flow signal was presented (RSS 100R Research Pneumotach System, Hans Rudolph, Shawnee, KS, USA).

RS were recorded simultaneously at posterior right and left chest (Rossi et al., 2000) (Fig. 1) using the LungSounds@UA interface. Two stethoscopes (Classic II S.E., Littmann ${ }^{\circledR}, 3 \mathrm{M}$, St. Paul, MN, USA), with a microphone (flat response between $20 \mathrm{~Hz}$ and $19 \mathrm{kHz}-$ TOM-1545P-R, Projects Unlimited, Inc. ${ }^{\circledR}$, Dayton, OH, USA) and a preamplifier circuit (Intelligent Sensing Anywhere ${ }^{\circledR}$, Coimbra, PT) in the main tube, were attached to the patient's skin with adhesive tape (Soft Cloth Surgical Tape, $3 \mathrm{M}$, St. Paul, MN, USA). The resulting analogue sound signals were further amplified and converted to digital by a multi-channel audio interface (MAudio ${ }^{\circledR}$ ProFire 2626, Irwindale, CA, USA). The signal was converted with a 24-bit resolution at a sampling rate of $44.1 \mathrm{KHz}$ and stored in .wav format.

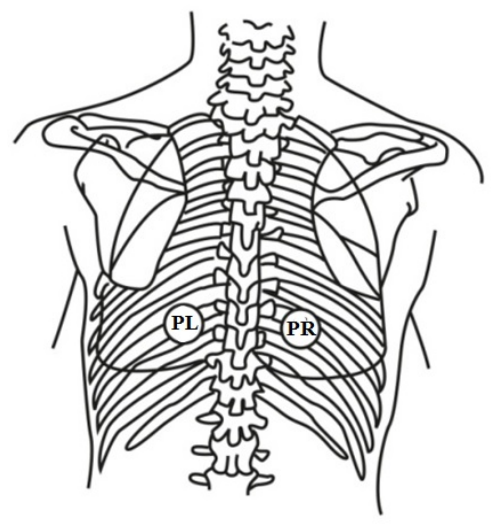

Figure 1. Chest locations, posterior right (PR) and posterior left $(\mathrm{PL})$, for respiratory sound recordings.

Spirometry was last performed according to standardised guidelines. Patients were then classified using the GOLD spirometric classification (Vestbo et al., 2013).

\subsection{Signal processing}

All RS files were processed using algorithms written in Matlab®R2009a (Mathworks, Natick, MA, USA). Breathing phases were automatically detected using the flow signals. To merge the detected breathing phases with sound files, signals were timed synchronised. Crackles were detected using a multialgorithm technique based on established algorithms (Quintas et al., 2013) and wheezes using an algo- rithm based on time-frequency analysis (Taplidou \& Hadjileontiadis, 2007). The mean number of crackles and wheezes per breathing phase was extracted. After excluding these adventitious sounds, normal RS were analysed based on the methodology proposed by Pasterkamp et al. (1996). The frequency of maximum intensity and mean intensity were determined within a frequency band of 300 to $600 \mathrm{~Hz}$.

\subsection{Statistical analysis}

Descriptive statistics were used to characterise the sample. Independent t-tests for continuous (age, body mass index and forced expiratory volume in one second, normal RS parameters), Mann Whitney U-tests for ordinal/not normal distributed (modified British Medical Research Council questionnaire, number of crackles, number of wheezes) and chisquare tests for categorical (GOLD classification, presence of wheezes) data were used to explore gender differences. The level of significance was set at 0.05 . Statistical analyses were performed using IBM SPSS Statistics version 20.0 (IBM Corporation, Armonk, NY, USA) and plots created using GraphPad Prism version 5.01 (GraphPad Software, Inc., La Jolla, CA, USA).

\section{RESULTS}

A total of 26 participants (13 females) were enrolled. No gender differences were noted regarding any of the socio-demographic or clinical characteristics (Table 1).

\subsection{Normal respiratory sounds}

The frequency of maximum intensity appeared to be slightly higher in females $(309.9-326.9 \mathrm{~Hz})$ than in males $(307.5-312.3 \mathrm{~Hz})$, mainly at posterior right chest (Fig. 2). However, no significant differences were found. The mean intensity of inspiratory normal RS was slightly higher in females (7.19-7.53dB) than in males (6.3-7.07dB) (Fig. 2). During expiration, the mean intensity was lower in females (5.18$5.43 \mathrm{~dB}$ vs. male 6.56-6.76dB), however, a significant difference was only found at posterior left $(\mathrm{p}=0.01)$ (Fig. 2).

Table 1. Socio-demographic, anthropometric and clinical characteristics of participants $(n=26)$.

\begin{tabular}{lcccc}
\hline Characteristics & Female* & Male* & \multirow{2}{*}{$p$} \\
\cline { 2 - 3 } & $\mathrm{n}=13$ & $\mathrm{n}=13$ & \\
\hline Age (years) & $63.3 \pm 9.4$ & $63.2 \pm 8.7$ & .99 \\
mMRC, M[IQR $]$ & $1[1,2]$ & $1[1,1]$ & .24 \\
BMI $\left(\mathrm{kg} / \mathrm{m}^{2}\right)$ & $26.5 \pm 4.9$ & $30.7 \pm 6.2$ & .07 \\
FEV $1 \%$ predicted $)$ & $91.1 \pm 13.8$ & $84.5 \pm 15.1$ & .26 \\
GOLD classification, $\mathrm{n}(\%)$ & & &
\end{tabular}


Mild

Moderate

$$
3(23 \%)
$$$$
4(31 \%)
$$

* Values are shown as mean \pm standard deviation unless otherwise indicated. mMRC, modified British Medical Research Council questionnaire; M, median; IQR, interquartile range;BMI, body mass index; $\mathrm{FEV}_{1}$, forced expiratory volume in one second; GOLD, Global Initiative for Chronic Obstructive Lung Disease.

\subsection{Adventitious respiratory sounds}

All patients had crackles both at posterior right and left. Wheezes were found in 17 patients at posterior right ( 7 females vs. 10 males; $p=0.205$ ) and in 12 patients at posterior left ( 6 females vs. 6 males; $p=1$ ). The median number of crackles (females 0.8-2.4 vs. males 2.9-4) and wheezes (females 0.3-0.4 vs. males 0.3-1.1) seemed to be higher in male patients (Fig. 3 ). Nevertheless, a significant difference was only observed in expiratory crackles at posterior right chest $(\mathrm{p}=0.04)$ (Fig. 3).
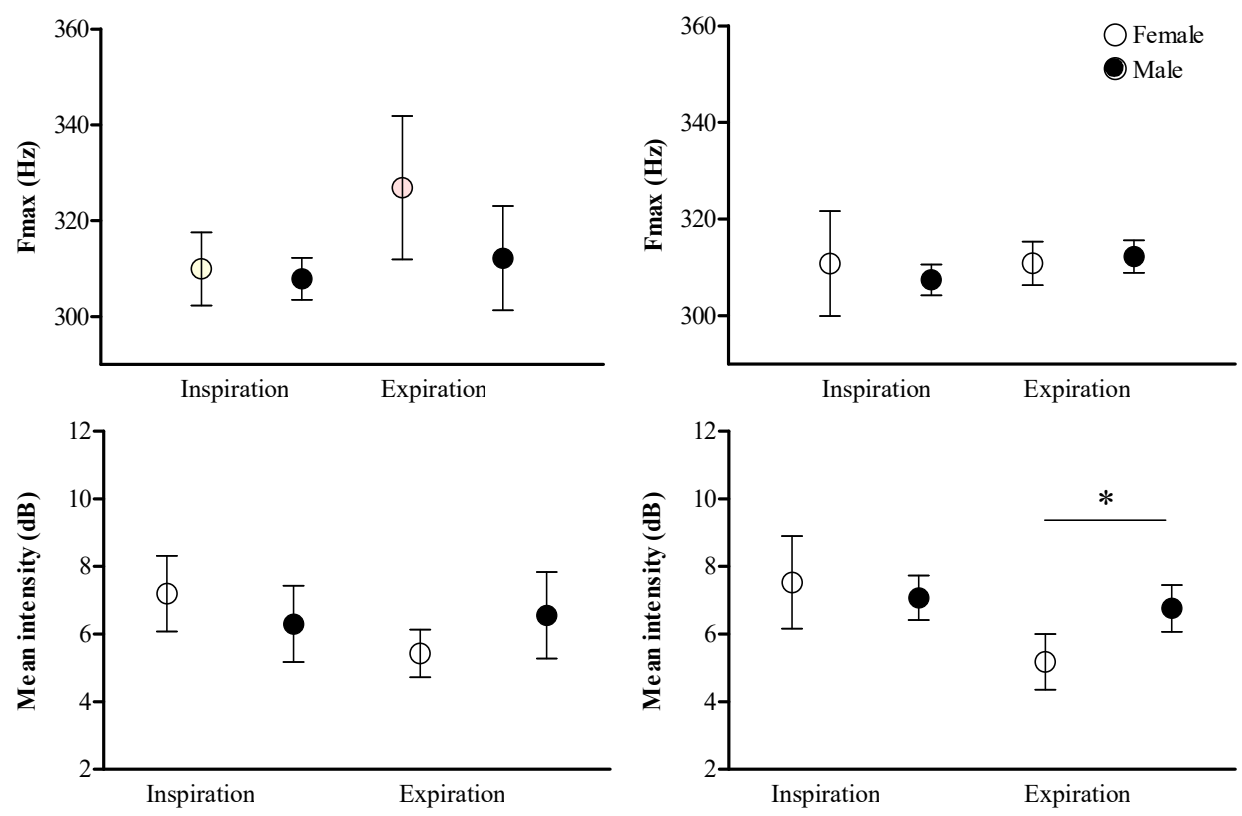

POSTERIOR RIGHT

POSTERIOR LEFT

Figure 2. Frequency of maximum intensity (Fmax - top panels) and mean intensity (bottom panels) per breathing phase of normal respiratory sounds at a frequency band of 300 to $600 \mathrm{~Hz}$ in female and male patients. Data are presented as mean and $95 \%$ confidence interval. Significant differences are identified with * $(\mathrm{p}<0.05)$.

\section{DISCUSSION AND CONCLUSIONS}

To the best of our knowledge this is the first study comparing computerised RS between female and male patients with COPD. Findings indicated that minor gender differences exist regarding normal and adventitious RS. This might be relevant for health professionals practice.

The frequency of maximum intensity appeared to be slightly higher in females than in males. This is in line with the study of Gavriely et al. (1995), which included healthy males and females, both nonsmokers and smokers. In this previous study it was found that females had higher frequency values compared to males, however significant differences were only found during inspiration (Gavriely et al., 1995). The mechanism behind these gender differ- ences is not well understood, but probably reflects the RS transmission through different thorax dimensions and configurations (Bellemare et al., 2003).

Intensity of RS were not significantly different between genders, with the exception of intensity of expiratory RS at posterior left, which was found to be lower in females. Gavriely et al. (1995) in their study with healthy males and females also did not find statistical significant differences in normal RS intensity. The significant difference found in the present study may be related with the airway volume mobilised during RS recordings. Despite the efforts in standardising airflow, volume was not standardised and it is known that the lung volume of adult female is typically $10-12 \%$ smaller than that of males, with the same height and age (Bellemare et 

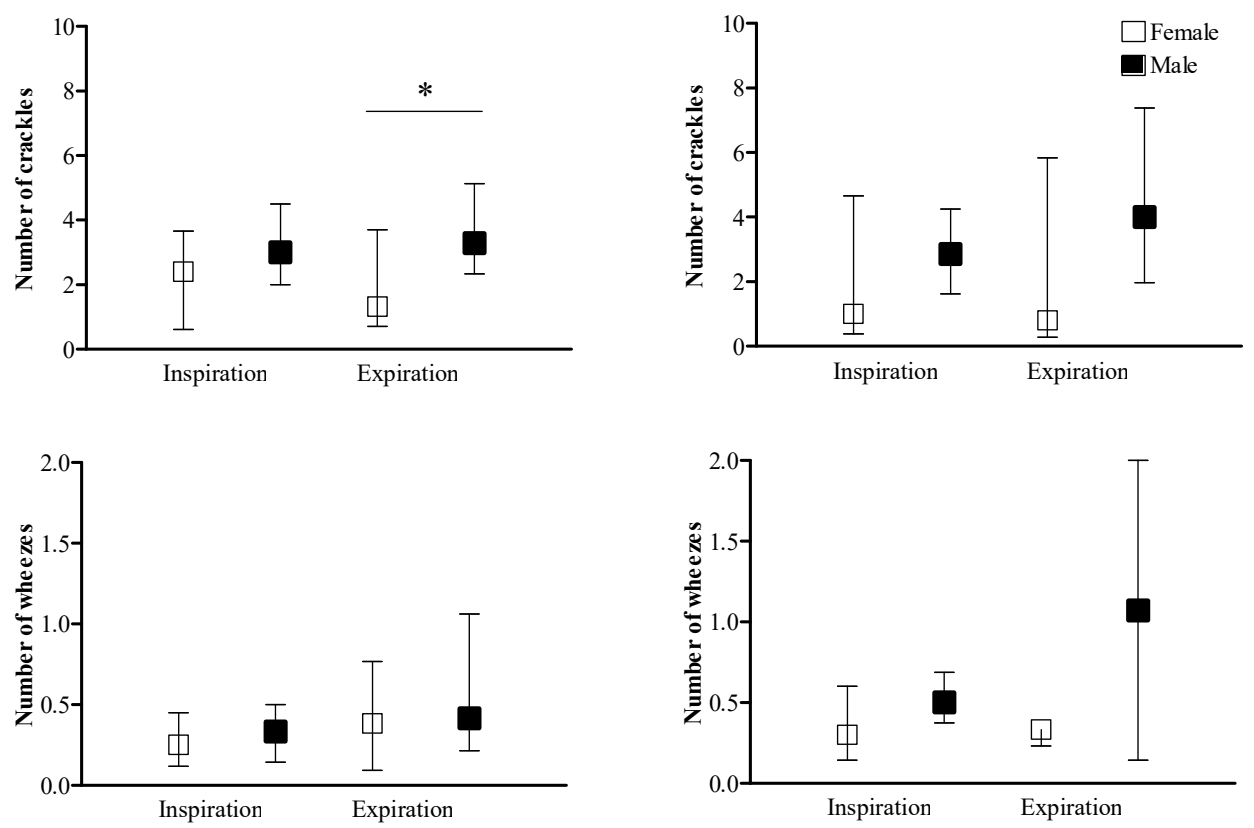

POSTERIOR RIGHT

POSTERIOR LEFT

Figure 3. Number of crackles (top panels) and wheezes (bottom panels) per breathing phase in female and male patients. Data are presented as median and interquartile range. Significant differences are identified with $*(\mathrm{p}<0.05)$.

Adventitious RS seemed to be more frequent in male patients. This finding may be related with the pathophysiology of COPD. Although airflow obstruction is the hallmark of COPD, the condition can develop on the basis of chronic bronchitis, emphysema or both. It has been found that, at all levels of disease severity, male patients have more extensive emphysema than females (Dransfield et al., 2010). Emphysema is related to the destruction of lung parenchyma and reduction of elastic recoil (Vestbo et al., 2013). These airway changes probably diminish the ability of airways to remain open, related with crackle genesis phenomenon (Vyshedskiy et al., 2009), as well as the critical flutter velocity, required to produce wheezes (Meslier et al., 1995).

This preliminary study has a number of limitations that need to be acknowledged. A small sample size of patients with early COPD was used and thus it was not possible to explore how the disease severity was related to the differences found. Nevertheless, data from this study may inform the sample size of future studies with more robust designs. Moreover, the present study focused in only four RS parameters. Future studies could investigate gender differences using other RS parameters, which may also have clinical relevance, such as median frequency of normal RS and wheezes occupation rate.

\section{CONCLUSIONS}

Findings suggest that minor differences exist between female and male patients with COPD regarding normal and adventitious RS. However, it is still unknown if these differences are clinically relevant. Further research with more robust designs is needed to explore other characteristics of computerised RS to draw definite conclusions.

\section{REFERENCES}

Bellemare F., Jeanneret A. \& Couture J. 2003. Sex differences in thoracic dimensions and configuration. Am J Respir Crit Care Med. 168(3):305-312.

Bohadana A., Izbicki G. \& Kraman S.S. 2014. Fundamentals of Lung Auscultation. N Engl J Med. 370(8):744-751.

Dransfield M.T., Washko G.R., Foreman M.G., Estepar R.S., Reilly J. \& Bailey W.C. 2007. Gender differences in the severity of CT emphysema in COPD. Chest. 132(2):464470.

Gavriely N., Nissan M., Rubin A.H. \& Cugell D.W. 1995. Spectral characteristics of chest wall breath sounds in normal subjects. Thorax 50(12):1292-1300.

Jácome C. \& Marques A. 2015. Computerized Respiratory Sounds in Patients with COPD: A Systematic Review. COPD 12(1):104-112. 
Meslier N., Charbonneau G. \& Racineux J.L. 1995. Wheezes. Eur Respir J. 8(11):1942-1948.

Pasterkamp H., Powell R.E. \& Sanchez I. 1996. Lung sound spectra at standardized air flow in normal infants, children, and adults. Am J Respir Crit Care Med. 154(2 Pt 1):424430.

Quintas J., Campos G., Marques A., 2013. Multi-algorithm Respiratory Crackle Detection. HEALTHINF - 6th International Conference on Health Informatics, Barcelona: SciTePress.

Rossi M., Sovijärvi A.R.A., Piirilä P., Vannuccini L., Dalmasso F. \& Vanderschoot J. 2000. Environmental and subject conditions and breathing manoeuvres for respiratory sound recordings. Eur Respir Rev. 10(77):611-615.

Taplidou S.A. \& Hadjileontiadis L.J. 2007. Wheeze detection based on time-frequency analysis of breath sounds. Comput Biol Med. 37(8):1073-1083.

Vestbo J., Hurd S.S., Agustí A.G., Jones P.W., Vogelmeier C., Anzueto A., et al. 2013. Global Strategy for the Diagnosis, Management, and Prevention of Chronic Obstructive Pulmonary Disease. Am J Respir Crit Care Med. 187(4):347-365.

Vyshedskiy A., Alhashem R.M., Paciej R., Ebril M., Rudman I., Fredberg J.J., et al. 2009. Mechanism of inspiratory and expiratory crackles. Chest. 135(1):156-164. 